\title{
Das Verstehen des pädagogischen Dialogs als Bedingung der Kriminalitätsbekämpfung
}

\begin{abstract}
Summary: The authors of this article investigate the possibility that the absence of pedagogical dialogues between educators and students can explain aspects of the incidence of crime, in particular acts of crime committed by young people. Based on this thesis and on basic principles of education, the relationship between language, communication and dialogue, as well as on the etiology and essential meaning, of the concept of "dialogue", the authors demonstrate that education is fundamentally an act of dialogue. The acknowledgement of this ontological fact will not only promote acceptable relationships and behaviour among the youth, but will also assist educators and social workers in their venture against the wave of criminality which is threatening to overpower the world.
\end{abstract}

\begin{abstract}
Резюме: Авторы настоящей статьи исследуют возможность объяснения определенных основных аспектов криминальности и/или подростковой криминальности по причине отсутствия педагогического диалога между воспитателями и воспитанниками. Исходя из выводов, как из важнейших признаков воспитания, отношения между языком, коммуникацией и диалогом, этимологией обозначения 'диалог', так и из характерных признаков диалога, авторы доказывают, что воспитание по природе своей диалогично. Признание данного методологического места и педагогической роли диалога в воспитании будет способствовать не только стремлению к приличию и приемлемому поведению среди (молодых) людей, но и помогать воспитателям, криминалистам и другим социальным работникам приводить людей к социально приемлемому поведению, на нашу общую пользу в нашей борьбе против волны преступности, которая наводнила мира.
\end{abstract}

Zusammenfassung: Die Autoren des vorliegenden Artikels (Understanding Pedagogical Dialogue as a Prerequisite for Counteracting Delinquency) untersuchen die Möglichkeit, gewisse Kernaspekte der Kriminalität bzw. Jugendkriminalität durch das Fehlen des pädagogischen Dialogs zwischen dem Erzieher und dem zu Erziehenden zu erklären. Ausgehend von Schlussfolgerungen sowohl aus den wesentlichen Merkmalen von Erziehung, dem Verhältnis zwischen Sprache, Kommunikation und Dialog, der Etymologie der Bezeichnung 'Dialog' als auch aus den charakteristischen Merkmalen von Dialog, weisen die Autoren nach, dass Erziehung ihrer Wesensart nach dialogisch ist. Die Anerkennung dieser ontologischen Stelle und pädagogischen Rolle von Dialog in der Erziehung wird nicht nur das Streben nach Anständigkeit und akzeptablem Verhalten unter (jungen) Leuten fördern, sondern auch Erziehern, Kriminologen, und anderen Sozialarbeitern helfen, Menschen zu einem sozial akzeptablen Verhalten zu führen, zum Vorteil von uns allen in unserem Kampf gegen die Kriminalitätswelle, die die Welt überschwemmt hat.

\section{Orientierung}

Aus akademischer Sicht sind wir uns durchaus im Klaren darüber, dass die vollkommene Zuverlässigkeit journalistischer Berichterstattung nicht immer garantiert werden kann (Snyman, 2007), aber wir sind trotzdem entsetzt über die vielen Berichte über die Kriminalitätswelle, die Südafrika und andere Weltteile überschwemmt (siehe Schipper, 2001, S. T19; Kotzé, 2003, S. 27; Thompson, 2004, S. 11; Seton-Smith, 2004, S. 16; Steyn \& Strydom, 2004, S. 340; Verbrugge, 2004; Böhm, 2005, S. 331-332; Louw, 2006, S. S13; Seleoane, 2006, S. 4; Van Niekerk, 2006, S. 4; Goosen \& Rossouw, 2006, S. 23; Nienhuis, 2006, S. 27; Van Hijum, 2006, S. 26; Enoch, 2006, S. 8; Fabbri, 2006, S. 6; Malan \& Meyer, 2006, S. 4; Hartle, 2006, S. 6; Olojede, 2006, S. 41; Thomson, 2007, S. 11; Rossouw, 2007, S. 217-218; Russo, 2007, S. 230; Malan, 2007, S. 18; Staude, 2007, S. 7; Geldenhuys, 2007, S. 1).

Allein die Existenz von diesen Berichten und Diskussionen über Gewalt, Verbrechen, Jugendkriminalität, widerspenstiges und unsoziales Verhalten sowie die Spekulationen in der 
Boulevardpresse über die eventuellen Gründe dafür deuten schon an, dass wir tatsächlich weltweit mit einem ernsten Problem in unseren Schulen und Gesellschaften konfrontiert sind.

Aufgrund der Forschung über das ubiquitäre Problem der Kriminalität bzw. Jugendkriminalität, Gewalt, Verbrechen, ungebändigtes und undiszipliniertes Verhalten im Allgemeinen in unserem eigenen Land Südafrika wie in vielen anderen Ländern (siehe auch Steyn \& Strydom, 2004, S. 339), kommen wir zu der Schlussfolgerung, dass das Problem zurückgeführt werden kann auf einen Zusammenbruch von Disziplin in der Gesellschaft im Allgemeinen und in Schulen im Besonderen. Tiefer gesehen könnte dieser Zusammenbruch dem Abbruch des pädagogischen Dialogs zwischen dem Erzieher und dem zu Erziehenden zugeschrieben werden.

\section{Methode}

In der vorliegenden Arbeit beabsichtigen wir, unsere Hypothese zu verteidigen, nach welcher der Zusammenbruch der Disziplin in der Gesellschaft im Allgemeinen sowie in Schulen im Besonderen im Wesentlichen auf einen Abbruch des pädagogischen Dialogs zwischen dem Erzieher und dem zu Erziehenden hindeutet. In dieser Darstellung verwenden wir drei Strategien: Zunächst entwickeln wir ein konzeptuelles Gerüst, in dem wir die Bedeutung von bestimmten Stichwörtern wie z.B. 'asoziale Verhältnisse', 'Kommunikation', 'Dialog', 'Erziehung' und 'authentische Erziehung' erläutern. Danach erfolgt eine Diskussion über die dialogische Art der Erziehung. Die Anwendungsphase besteht darin, dass wir die bis dahin gesammelten Befundnisse verwenden, um den Prozess und die Folgen des Abbruchs des pädagogischen Dialogs zu erklären. Im Schlussteil der Arbeit befassen wir uns mit der Rechtfertigung unserer Hypothese und dem Erstellen von Empfehlungen.

Das Ziel unserer Forschung war in erster Linie, das Problem der Störung des pädagogischen Dialogs zu verstehen. Zum Verstehen der verschiedenen ontischen Aspekte des Problems haben wir zunächst die phänomenologische Methode benutzt, d.h. wir haben uns auf die Ontizität der Aspekte des pädagogischen Dialogs konzentriert und danach die Methode der konzeptuellen Analyse durchgeführt. Die Untersuchung wurde fortgesetzt mit einer interpretivistisch-hermeneutischen Diskussion über Dialog und Abbrüche eines pädagogischen Dialogs. Nachdem wir die nötigen Maßnahmen getroffen hatten, um die Gefahr eines naturalistischen Trugschlusses zu vermeiden (Honderich, 2005, S. 642), verwendeten wir die von uns aufgedeckten ontischen Merkmale des pädagogischen Dialogs, um die Wesensmerkmale eines authentischen pädagogischen Dialogs darzustellen. Danach haben wir in folgender Weise argumentiert: Wenn eine bestimmte Interaktion bzw. ein Gedankenaustausch zwischen dem Erziehenden und dem Edukanden nicht diese besonderen ontischen Merkmale offenbart, müsste daraus geschlossen werden, dass es sich um einen Abbruch des pädagogischen Dialogs handelt. Unserer Meinung nach könnte solch ein Abbruch des Dialogs zu asozialem Verhalten und Jugendkriminalität führen.

Die aktive Erarbeitung von Strategien zur praktischen Bekämpfung von Verbrechen und Jugendkriminalität überlassen wir den betreffenden Fachleuten, wie z.B. schulischen Lehrkräften, Beamten von Hafteinrichtungen, Sozialarbeitern usw. Auch der Entwurf einer Politik zur Bekämpfung von Kriminalität bzw. Jugendkriminalität fällt in den Zuständigkeitsbereich von politischen Entscheidungsträgern der unterschiedlichen Bereiche. Da die vorliegende Forschungsarbeit sich nicht zum Ziel setzt, Empfehlungen zu erstellen für die Wiederherstellung des pädagogischen Dialogs in Situationen, in denen der pädagogische Dialog schon gestört worden ist, gibt es keine Hinweise, Strategien und Ratschläge für eventuelle Verfahrensweisen zur Wiederherstellung von unterbrochenen bzw. zerstörten Dialogen.

Weiterhin möchten wir darauf aufmerksam machen, dass das in der vorliegenden Arbeit referierte Problem sich nicht experimentell untersuchen lässt. Aus ethischen Gründen wäre es nicht 
zu rechtfertigen, zwecks eines Vergleiches einige Kinder und junge Leute einer 'schlechten', 'ineffizienten' und 'ungesunden' 'Erziehung' (sollte eine derartige contradictio in terminis provisorisch gestattet sein) und andere einer 'guten', 'effektiven' und 'gesunden' 'Erziehung' (hier pleonastisch verwendet) auszusetzen.

\section{Konzeptuelles Gerüst}

\section{Antisoziales Verhalten}

Antisoziales Verhalten verweist im Allgemeinen auf jegliches Verhalten, das die Verletzung von sozialen Normen, schädliche Handlungen gegenüber anderen oder beides - aggressive Handlungen wie Diebstahl, Vandalismus, Brandstiftung, Schulschwänzen, Herausforderung von Autorität und Lügen - bewirkt (Slade \& Schneider, 2001, S. 4). Rottschaefer (2000, S. 263) ist davon überzeugt, dass antisoziales Verhalten nicht nur unethisch, sondern auch unmoralisch ist, da solche Handlungen sowohl an der Enthumanisierung und Zerstörung von intellektuellen, kreativen, praktischen und sozialen Leistungen als auch an der Störung gesellschaftlicher Wachstumsprozesse, an sozialer Ungerechtigkeit und der Beeinträchtigung von moralischen Rechten beteiligt sind. Seiner Ansicht nach (Rottschaefer, 2000, S. 271) sind antisoziale Absichten und Aktionen solche, die das Leiden oder die Verletzung eines anderen Menschen zum Ziel haben. Obwohl es viele kriminologische und soziologische Theorien über die Ursachen und den Umfang kriminellen Verhaltens gibt, konzentrieren wir uns nur auf den Abbau oder den Verlust des pädagogischen Dialogs als eine potenzielle Ursache. ${ }^{1}$

\section{Dialog und Erziehung}

Trotz der Tendenz, Dialog als Heilmittel für allerlei Probleme vorzuschlagen, einschließlich Irrationalität, multikulturellen Streits, ineffizienter Sozialisierung, der Gestaltung einer bürgerlichen Gesellschaft, postmoderner Ethik und der Frage, was es hieße, 'menschlich zu sein' (Lefstein, 2006, S. 1), sollte er weder aufgewertet noch als das endgültige pädagogische Universalmittel für gesellschaftliche Krankheiten dargestellt werden. Und im Falle verfehlten Dialogs sollte auch nicht angenommen werden, dass die Lösung zu Problemen im verharrenden Dialogführen besteht (Burbules, 2003, S. 195).

Ohne Sprache kann Dialog nicht zustande kommen. Sprache als Werkzeug zum Denken und zur Kommunikation (Department of Education, 2007, S. 15) und Dialog als expressiver Modus der Sprachverwendung können als Korrelate des Menschseins überhaupt beschrieben werden. Sprache ist ein exklusiv und universal menschliches Phänomen und eines der bemerkenswertesten Lebensmerkmale, das Menschen von Tieren unterscheidet (Potgieter, 1991, S. 229). Dialog ist das Gewand, in dem wir den Inhalt unserer Gedanken vor anderen Menschen zur Schau tragen (Hendricks, 1988, S. 38). Daher ist Dialog nicht blo $\beta$ eine 'Form' der Kommunikation, sondern im Wesentlichen Kommunikation selbst.

In einem Erziehungskontext deutet die Etymologie des Wortes 'Kommunikation' darauf hin, dass der Edukator und der Edukand sich immer in einem spezifischen Verhältnis zueinander befinden und daher wesentlich miteinander verbunden sind. Weiterhin setzt Kommunikation voraus, dass es einen Zuhörer gibt, ob Peer oder Lehrer, und es ist dieser Dialog, die reziproke Handlung von Hören und Reagieren, der der Erziehung zugrunde liegt.

Axiale Kodierung (De Vos, 2002; Ertmer, 1997; Punch, 1998) und eine heuristische Untersuchung der Definitionen und Erläuterungen des Wortes 'Dialog' decken die wesentliche 
Voraussetzung für einen effizienten Dialog auf, nämlich die Erfüllung folgender Normen: Dialog sollte Menschen als sinnsuchenden und sinnfindenden Wesen helfen, Sinn im Leben und Umfeld (Umwelt) wie im Gewissen, in Emotionen und Spiritualität (Eigenwelt) zu finden. Die Handlungen der Dialogteilnehmer sollen Zielausrichtung und Intentionalität ausdrücken und dabei gegenseitige Motivation, Belebung, Stärkung, Zutrauen, Engagement, Assoziierung (Zusammensein) und Begegnung anregen. Dialog soll charakterisiert sein durch reziprokes Engagement (Fr. engager verpflichtet/gezwungen sein, etwas zu tun). Die Teilnehmer sollen gegenseitig erreichbar, verfügbar, tolerant und treu sein; ihre Begegnungen sollen sowohl eine Atmosphäre von Vertrautheit, Gleichzeitigkeit, Zugehörigkeit, freiwilliger Interaktion, aufrichtiger Begegnung (Nähe) und transparenter Teilnahme (Kooperation/ Zusammenarbeit) als auch Einverständnis, Gleichmut, gegenseitige Anerkennung und selbstlose Reziprozität kreieren und aufrechterhalten. Der Gehalt und die Sinnstiftung des Dialogs sollen appellierend und beeinflussend sein, ausgerichtet auf sinnvolle, konstruktive Antworten, ehrliche Selbstdarstellung und Bewusstseinsbildung. Dialog soll Einigkeit der Teilnehmer erstreben und sich an ihren Entscheidungen orientieren. Weiterhin soll Dialog Selbstbestimmung und Charakterisierung anerkennen, pflegen und fördern; er soll auf Können basiert und auf offene, transparente Wissensteilung ausgerichtet sein, gegründet auf wahrheitsliebender Würdigung, normativer Rationalität, ehrlicher Interpretation und gegenseitig vorteilhafter Verhandlung und Konsultation.

Diese Normen sind hauptsächlich ziel- und weniger handlungsorientiert, denn unser Ziel mit dieser Forschung war in erster Linie, das Problem zu verstehen und grundsätzliche Richtlinien vorzuschlagen. Demnächst ist es die Pflicht von u.a. politischen Entscheidungsträgern, den entsprechenden Fachleuten, wie z.B. Erziehern anzudeuten, wie sie z.B. im Klassenzimmer vorgehen sollten, um Edukanten behilflich zu sein in ihrem Sinnfindungsprozess in Bezug auf ihr persönliches Leben, ihre soziale Umgebung, ihr Gewissen, ihre Emotionen und Spiritualität. Aus diesem Grund ist es erforderlich, diese abstrakten Konzepte in konkrete Vorschläge umzusetzen und dabei anzudeuten, wie diese Zielsetzungen in der Praxis gehandhabt werden sollten, um sie dem Bildungspraktiker verwendbar zu machen.

Wenn das Ziel, der Gehalt und die Relation/Form des Dialoges alle in Harmonie miteinander funktionieren, etabliert sich authentischer Dialog. Wenn sie alle effizient funktionieren, intensiviert sich der Dialog zur Kommunikation.

\section{Erziehung}

Eine Analyse von Wörterbuch-Definitionen von Erziehung (Bosman, Van der Merwe \& Hiemstra, 2004; Chevalley, Abel \& Marquerite, 1980; Eksteen, 1984; Finch, o.J;; Foreman, 1975; Kritzinger, 1990; Kritzinger, Schoeman \& Cronjé, 1996; Postma, 1992; Schoonees, Swanepoel, Du Toit \& Booysen, 1975; Simpson, 1980; Strelen \& Gonin, 1970; Sykes, 1993; Terblanche \& Odendaal, 1976; Oxford English Dictionary, 1993a \& b; Van Rensburg \& Landman, 1995) und ein Vergleich der Ergebnisse mit den Definitionen wie Erklärungen und Rechtfertigungen desselben Begriffes, verkündet von einer Anzahl wohlbekannter Philosophen, Psychologen und Erzieher (u. a. Van Rensburg \& Landman, 1995 und Burbules, 1993), decken die wesentlichen Eigenschaften von Erziehung auf. Wenn eines oder mehrere dieser Wesensmerkmale oder Strukturelemente in einem Gedankenaustausch zwischen einem Edukator und einem Edukanden fehlen, oder wenn eines von ihnen fehlen oder misslingen würde, muss man bezweifeln, ob Erziehung überhaupt stattgefunden hat. Die Wesensmerkmale oder Strukturelemente der Erziehung können daher als strukturelle Richtlinien für authentische Erziehung aufgefasst werden. 


\section{Authentische Erziehung}

Im Rahmen der Beweisführung zugunsten von 'Strukturnormen' kann authentische Erziehung nur unter bestimmten Verhältnissen stattfinden, nämlich einerseits, wenn die Interaktion zwischen einem Edukator und einem Edukanden eine erzieherische, soziale und dialogische Beziehung darstellt, und andererseits, wenn es eine bewusste, intentionale, zielgerichtete, wirksame und dialogische Teilnahme bzw. Beteiligung, aber auch eine Intervention seitens eines Edukators (Erwachsenen) in das Leben eines abhängigen, hilfsbedürftigen Edukanden gibt. In letzterem Fall ist das Ziel der Intervention, den Edukanden zu einer normativen Sinnfindung und Erschließung seiner Lebensaufgabe innerhalb einer religiös bedingten kulturellen und zivilisierten Umgebung zu führen. (Nach der 'Struktur und Religion / Weltanschauung als lebensbestimmende Richtung'Argumentation müsste die spezifische Religion oder Lebensansicht definiert werden.)

Erziehung erzielt demnach die Leitung und Begleitung des Edukanden seitens des Edukators und der zugehörigen Gesellschaft, mit der der Edukand sich identifiziert, auf eine anständige, vorbildliche und instruktive Weise, in Richtung physischen, geistigen, moralischen, ästhetischen und spirituellen Erwachsenseins als Selbstverwirklichung, den erhabensten und wertgeschätzten Tugenden (Überzeugungen, Sitten, Werten, Haltungen und Erfordernissen der Wohlanständigkeit) entsprechend. Der Edukand, der auf dem Weg zum Erwachsensein ist, sollte (von dem Erwachsenen) geführt, begleitet und gestärkt werden, ständig das Image authentischen Erwachsenseins und eine selbständige Urteilsfähigkeit zu entfalten, so dass sein vom Erwachsenen Geführtsein allmählich redundant werden sollte. Nur durch engagierten, konstruktiven Dialog kann dieses Ziel erfolgreich erreicht werden (Langeveld, 1968, S. 47; Ten Have, 1970: passim; o.J., S. 59; 1973, S. 3).

Durch Erziehung erstreben Erwachsene die Instruierung von Edukanden in Bezug auf die Entwicklung und Verbesserung ihrer kritischen Lebens- und Sozialfähigkeiten, wie z.B. Fähigkeiten des Entscheidungsvermögens, der Verweigerung, der kritischen Analyse und des systematischen Urteils, vor allem des gesunden Urteilsvermögens (einschließlich des Vermögens, zwischen kulturell und sozial akzeptablem bzw. inakzeptablem Verhalten zu unterscheiden). Dies kann auch nur durch engagierten, konstruktiven Dialog erfolgreich erreicht werden.

Erziehung ist nicht die alleinige Verantwortung des Erwachsenen/Lehrers/Edukators. Zur Erreichung eines angemessenen Erziehungsstandes sollte der Edukand, so wie er älter wird, zunehmend Mitverantwortung für seine Erziehung übernehmen. In seiner Progression zum Erwachsensein sollte der Edukand lernen, den zunehmenden Lebensanforderungen gewachsen zu sein. Für den Edukanden heißt Erziehung die Einbindung in Aktivitäten der Vermittlung und Aneignung, die dem Fortschreiten zum Erwachsensein dienen.

\section{Die dialogische Natur von Erziehung}

Die fundamentale Koexistenz von Menschen (vgl. Langeveld (1968, S. 158), Heidegger (1977, S. 161)), verweist nicht nur auf das immerwährende existenzielle Bedürfnis eines Menschen nach einem anderen Menschen, sondern auch auf den dialogischen Bereich und Umfang von agein, d. h. auf die Frage: wer richtet den Appell und wer reagiert auf den Appell? Im Geburtsmoment verlässt das Kind den Zufluchtsort der mütterlichen Körperlichkeit auf immer. Er verkündet seine Ankunft in dieser Welt mit der ersten Äußerung, dem Geburtsschrei, durch den er sein lebenslängliches Angewiesensein auf seine Mitmenschen für seine eigene Menschwerdung zu erkennen gibt. Der Geburtsschrei ist sowohl die fundamentalste Verlautbarung menschlicher Existenz als auch der fundamentalste Appell um Hilfe. Diese appellative Erklärung bzw. Frage repräsentiert nicht nur die 
prototypischen Anfänge erzieherischen Dialogs, sondern auch die wesentlich dialogische Art des erzieherischen Handelns (Potgieter, 2006). Der Edukand lebt nicht nur in offener Kommunikation mit der Welt, sondern auch in einer Interkommunikation mit den Menschen, mit denen er diese Welt teilt (Smit, 1977, S. 262; Groothoff, 1964, S. 28; Levinas, 1969, S. 175).

Das Betragenlernen in einer kulturell und sozial akzeptierbaren Weise dürfte und sollte deswegen nicht nur dem Edukanden oder dem Schicksal überlassen werden. Gereifte Erwachsene sollten die Edukanden das richtige Benehmen lehren. Damit diese pädagogische Assoziierung und das bildende Verhältnis authentisch wirken können, ist Dialog unbedingt erforderlich. Dialog, aktualisiert durch den Gebrauch von Sprache, ist demnach die conditio sine qua non für jedes pädagogische Verhältnis. Bandura (2002, S. 109) befürwortet diese Ansicht mit der Behauptung, dass eine der Funktionen von Sprache in der 'verpersönlichenden Verantwortlichkeit zur Humanisierung von anderen Menschen' besteht. Ertmer und Newby (1996, S. 15 \& S. 18) betonen die Rolle des pädagogischen Dialogs als ein Werkzeug der Reflektierung, als eine spezielle Form des Denkens, das unbedingt erforderlich ist für die Transformation des Wissens, das der Edukand in pädagogischen Begegnungen gewinnt. Lefstein (2006, S. 3) behauptet, dass der Edukand zum 'Dialog gedrängt wird durch das Bewusstsein seiner eigenen Ignoranz und seinen Wissensdurst', während Freire (1970, S. 47), und Burbules und Berk (1999, S. 9) argumentieren, eine der Zielsetzungen pädagogischen Dialogs wäre die Kritik sozialer Interaktionen, sozialer Verhältnisse, sozialer Traditionen und sozialer Dynamik, um des Edukanden kulturelle Entwicklung zur Freiheit hin zu erwirken. Diese Autoren betrachten Dialog als eine der wichtigsten pädagogischen Methoden zur Förderung dieser Art von Kritik (Burbules \& Berk, 1999, S. 19).

Die Voraussetzung für einen erfolgreichen Dialog ist die Erfüllung bestimmter Vorbedingungen. Typischerweise schlie $\beta$ t das die Bereitwilligkeit der Dialogpartner ein, sich physisch wie geistig in eine kommunikative Situation zu begeben, an einem Dialog teilzunehmen unter den Prämissen, dass beide Partner das Recht haben, ihre Meinung offen zu sagen und auch die Pflicht, dem anderen zuzuhören. In allen Situationen eines pädagogischen Dialogs sollten die erwachsenen Partner ein Vorbild akzeptablen Verhaltens darstellen, das ebenfalls von den jungen Teilnehmern praktiziert werden sollte. Ein Dialog ist viel mehr als ein bloßer Austausch von Worten. Im Grunde genommen ist es eine bedeutungsvolle Bindungskraft im Leben, Denken und Handeln von jungen Menschen. In dieser Hinsicht ist Martin Bubers Lehre vom Ich-Du von besonderer Bedeutung.

\section{Die Folgen eines Fehlens des pädagogischen Dialogs}

Gestörter oder fehlender pädagogischer Dialog kann verstanden werden als eine Funktion der Erziehung, die ihr Ziel verfehlt hat. Zum Verstehen der Ursachen für diese Störung wollen wir uns zunächst befassen mit einer Beobachtung und Untersuchung der asozialen, enthropischen Art der Relationen in den Fällen, wo Beziehungen zusammengebrochen sind. Weiterhin soll die Untersuchung sich konzentrieren auf die Formen, Methoden und Bedeutungen, die von einem besonderen Fall gestörten pädagogischen Dialogs exemplifiziert werden, wie z.B. Krankheitsvortäuschung, Schulschwänzen, gewalttätiges Verhalten, beleidigende Sprache, Diebstahl und Selbstverstümmelung, denn das sind Manifestationsarten asozialen Verhaltens in der wirklichen Welt. Durch Beobachtung und Untersuchung von den Relationen, Formen, Methoden und Bedeutungen, die ein besonderer Fall gestörten pädagogischen Dialogs darstellt, wird es möglich, die zu einem jeden Fall beitragenden Faktoren aufzudecken. Das Recherchieren dieser Einzelheiten ermöglicht es einem, ihnen eine spezifische Relevanz zu verleihen und auf diese Weise die zu Grunde liegenden Intention(en), Ziel(e) und die Zweckmäßigkeit dieses besonderen Falles asozialen Verhaltens zu bestimmen. Schließlich gewinnt man Verständnis dafür, so dass Gründe für die pädagogische Störung in dem besonderem Fall einsichtig gemacht werden können. 
Die Störungen pädagogischen Dialogs können in drei Hauptkategorien eingeordnet werden, nämlich Störungen herbeigeführt vom (1) Edukator bzw. vom (2) Edukanden, oder aber von (3) beiden Parteien. Zunächst betrachten wir die vom Edukator herbeigeführte Störung des Dialogs.

Das moderne Familienleben ist zunehmend charakterisiert durch die Abwesenheit der Eltern wegen Arbeitsanforderungen, Berufsreisen, Elternentfremdung, Ehescheidung, HIV/AIDSSterbefälle (wie in Afrika) und anderen Verhältnissen, die sinnvolle pädagogische Interaktionen zwischen Eltern und Kind (als Edukanden) verhindern. Demnach ist die zunehmende Störung von Inhalt und Form des Eltern-Edukanden-Dialogs vorauszusetzen. Dass Eltern dem Edukanden nicht mehr ohne Weiteres zur Verfügung stehen, ist im Grunde genommen die Erodierung der wesentlichen pädagogischen Merkmale sowohl der Verfügbarkeit, Nähe, Zugehörigkeit, freiwilliger Interaktion und transparenter Teilnahme als auch des Einverständnisses, des Gleichmuts, der gegenseitigen Akzeptanz und selbstloser Reziprozität. Eltern teilen nicht mehr denselben ZeitRaum-Bereich mit dem Edukanden, wodurch das wesentliche pädagogische Merkmal der Gleichzeitigkeit erodiert wird. Dies führt zu fehlender Interaktion zwischen Eltern und Edukanden, und weiterhin zum Verlust der Möglichkeit, eine Atmosphäre dialogisch-diagogischen Austausches sowie einer aufrichtigen Begegnung zu kreieren (Pretorius, 1999, S. 141-144). Wenn die wesentlichen Eigenschaften der dialogischen Form derartig erodiert bzw. unterminiert werden, können sie nicht wirksam sein. Wenn keine dialogische Interaktion stattfindet, und wenn keine dialogisch-diagogische Atmosphäre der Begegnung besteht - beides wesentliche Merkmale der dialogischen Form -, kann auch keine pädagogische Wertschätzung, Genehmigung, Anerkennung oder Konversation mehr stattfinden. Schließlich würde das heißen, dass die Erfüllung der wesentlichen Merkmale des dialogischen Gehalts, nämlich normative kritische Rationalität, Verantwortlichkeit oder Selbstbestimmung, nicht mehr möglich wäre.

Der Scheitern des pädagogischen Dialogs seitens des Edukanden könnte freiwillig oder unfreiwillig stattfinden. Bekanntlich tendieren Edukanden, die an selektivem Mutismus, einem sozialen Angstzustand, leiden, dazu, den pädagogischen Dialog unfreiwillig bzw. unabsichtlich zu unterbrechen.

Ein Beispiel freiwilligen Unter- oder Abbrechens des pädagogischen Dialogs ist die adoleszente Widerspenstigkeit. Manifestationserscheinungen abweichenden Benehmens sind u.a. Angst und Ängstlichkeit, die Entscheidung, Straßenkind zu werden, Schulschwänzen, Krankheitsvortäuschung, Teilnahme an kriminellen Handlungen, Drogenmissbrauch, Herausforderung der Autorität der Erzieher, Eigensinn und Halsstarrigkeit sowie Launenhaftigkeit (Du Toit, 2006). Im Grunde genommen beinhaltet adoleszente Angst ein Gefühl der Einsamkeit, das durch die Trennung vom liebevollen Verbundensein entsteht. Jedesmal, wenn dem abhängigen, hilfsbedürftigen Adoleszenten die liebevolle Verbundenheit fehlt, erlebt er es als eine Bedrohung seiner Existenz, als Angsterregung. Bei schlimmer werdenden Verhältnissen wird der Adoleszent umso empfindlicher, und dementsprechend nimmt seine 'Bereitschaft, Elend zu erleiden' zu. Als junger Mensch fehlt ihm die Kraft und Reife zur Wiederherstellung authentischen Kontakts, so dass er sich stattdessen entweder in sich selbst zurückzieht oder, in einem Versuch der Selbstwehr, auf die Außenwelt fixiert, oder aber aus Angst um das Nichterscheinen 'nach außen hinguckt'. Das äußerliche Verhalten des Edukanden kann daher entweder schüchtern und zaghaft ('Fluchtstellung') oder gleichgültig, unhöflich, impulsiv oder negativ sein ('Abwehrstellung').

Aus des Adoleszenten Erfahrung der mangelhaften pädagogischen Betreuung (aufgrund pädagogischer Fehler oder des Adoleszenten eigener Hypersensibilität) entsteht eine Furcht vor Dialog, da dieser ihn schon wieder verletzen könnte und daher entweder vermieden oder zerstört werden müsste. Auf diese Weise etabliert sich ein Zyklus: Die Furcht vor Kontakt sperrt die Kontaktmöglichkeit und wird praktisch zu einer objektiven Gefährdung der oben genannten 
Verbundenheit des Seins (Du Toit, 2006; Kirsten, 2006; siehe weiter Black \& Uhde, 1992, S. 10901094; Black \& Uhde, 1994; Black \& Uhde, 1995; Black, 1995; Black, 1996; Black, Leonard \& Rapoport, 1997).

Das dritte Beispiel bezieht sich auf die Störung des pädagogischen Dialogs von beiden Parteien, dem Edukator wie dem Edukanden. Vorfälle, bei denen der pädagogische Dialog absichtlich von dem Edukanden unterbrochen wird, sind selten monokausal durch den Edukanden bedingt. In der Mehrheit solcher Fälle kann die diesbezügliche Entscheidung des Edukanden auf elterliche/erzieherische Kontrolle zurückgeführt werden (Du Toit, 2006; Kirsten, 2006). In diesem Fall kann der gestörte pädagogische Dialog interpsychischem Konflikt zugeschrieben werden (siehe weiter Department of Health and Human Services, Administration on Children, Youth, and Families, 2001; Knutson, DeGarmo \& Reid, 2004, S. 187f.).

\section{Schlussfolgerungen und Empfehlungen}

Basierend auf den drei Strategien zur Verteidigung unserer Prämisse, d.h. die These, die wir in dem einführenden Teil der vorliegenden Arbeit dargeboten haben, kommen wir nun zur Schlussfolgerung, dass - unserer Meinung nach - die Validität jener Schlussfolgerung belegt worden ist. Wir haben tatsächlich festgestellt, dass eine Reihe von Faktoren eine bedeutungsvolle Rolle in dem Entstehen (jugendlichen) asozialen Verhaltens spielen kann und tatsächlich spielt. Unsere Untersuchung bestätigt also die Möglichkeit, dass das Scheitern des pädagogischen Dialogs zwischen Edukator und Edukand bestimmte Kernaspekte dieses Phänomens erklären könnten.

Ausgehend von Beispielen jugendlichen asozialen Verhaltens sowie Schlussfolgerungen in Bezug auf die wesentlichen Eigenschaften der Erziehung, das Verhältnis zwischen Sprache, Kommunikation und Dialog, die Etymologie des Wortes 'Dialog' und die wesentlichen Eigenschaften von Dialog, haben wir vorgeführt, dass Erziehung ihrem Wesen und ihrer Absicht nach dialogisch ist. Wenn pädagogischer (erzieherischer) Dialog aus irgendeinem Grund unterbrochen wird, sollte man daraufhin disruptives, undiszipliniertes, verbrecherisches und asoziales Verhalten erwarten, unabhängig davon, ob das asoziale Verhalten aus dem Abbruch des Dialogs durch den Edukator, den Edukanden oder die beiden resultiert.

Die Erforschung der wesentlichen Strukturen von Dialog-in-Erziehung führte zu dem Ergebnis, dass die Korrelation der Kategorien Ziel, Gehalt und Form bezüglich der Wesensart von Dialog zwei zu Grunde liegende Sequenzen aufdeckt, nämlich einerseits eine Anwendungssequenz (d.h. wie authentischer pädagogischer Dialog erfolgreich angewandt, durchgeführt und erreicht werden kann) und andererseits eine diagnostische Sequenz (d. h. wie festzustellen ist, wo und in welchem Ma $\beta$ pädagogischer Dialog gestört ist).

Die Anerkennung dieser ontologischen Stelle und agogischen Rolle von Dialog in Erziehung wird nicht nur das Streben nach Anständigkeit und akzeptablem Verhalten fördern, sondern auch Kriminologen, Erziehern, Friedensstiftern, Sozialarbeitern, Heilpraktikern und lebensmutigen Menschen helfen, die Welt besser bewohnbar und humaner zu machen (Orr, 1991, S. 53 \& S. 55). Ein gebührender Fokus auf den pädagogischen Dialog wäre ein Ansatz zur Stärkung der bestehenden humanitären Versuche, soziale Systeme zu etablieren und zu erhalten, die anteilnehmendes Verhalten (Bandura, 2002, S. 116) pflegen und demzufolge uns allen in unserem Kampf gegen Kriminalität helfen würden.

Wir möchten daher empfehlen, dass Erziehungspraktiker, einschließlich politischer Entscheidungsträger, die Umsetzung der von uns aufgedeckten sechs ontischen Strukturelemente des pädagogischen Dialogs in die Praxis überlegen sollten. 
Pädagogischer Dialog hilft Menschen als sinnsuchenden und sinnfindenden Wesen Sinn im Leben und Umfeld (Umwelt) wie im Gewissen, in Emotionen und Spiritualität (Eigenwelt) zu finden. Die Handlungen der Dialogteilnehmer drücken Zielausrichtung und Intentionalität aus und dabei regen sie gegenseitige Motivation, Belebung, Stärkung, Zutrauen, Engagement, Assoziierung (Zusammensein) und Begegnung an. Dialog offenbart sich durch reziprokes Engagement (fr. engager - verpflichtet/gezwungen sein, etwas zu tun). Teilnehmer sind gegenseitig erreichbar, verfügbar, tolerant und treu; ihre Begegnungen sind sowohl eine Atmosphäre von Vertrautheit, Gleichzeitigkeit, Zugehörigkeit, freiwilliger Interaktion, aufrichtiger Begegnung (Nähe) und transparenter Teilnahme (Kooperation/ Zusammenarbeit), und es geht auch darum, Einverständnis, Gleichmut, gegenseitige Anerkennung, selbstlose Reziprozität aufrechtzuerhalten. Der Gehalt und die Sinnstiftung des Dialogs appellieren und beeinflussen, ausgerichtet auf sinnvolle, konstruktive Antworten, ehrliche Selbstdarstellung und Bewusstseinsbildung. Dialog erstrebt Einigkeit der Teilnehmer und orientiert sich an ihren Entscheidungen. Weiterhin anerkennt, pflegt und fördert Dialog Selbstbestimmung und Charakterbildung; er basiert auf Können und ist ausgerichtet auf offene, transparente Wissensteilung, und er ist gegründet auf wahrheitsliebender Würdigung, normativer Rationalität, ehrlicher Interpretation und gegenseitig vorteilhafter Verhandlung und Konsultation.

Danksagung: Herrn Professor Dr. Gerhard Zecha von der Katholischen Universität Salzburg in Österreich danken wir für seine wertvollen Ratschläge.

\section{Literaturverzeichnis}

Bandura, A. (2002): Selective Moral Disengagement in the Exercise of Moral Agency. In: Journal of Moral Education. Vol. 31. No. 2, S. 101-119.

Black, B. (1995): Fluoxetine for (s)elective mutism [reply to letter]. In: Journal of the American Academy of Child \& Adolescent Psychiatry, 34(6), S. 702-703.

Black, B. (1996): Social anxiety and selective mutism. In: American Psychiatric Press Review of Psychiatry, Volume 15. Washington: American Psychiatric Press, Inc., S. 469-496.

Black B.; Leonard H.L.; Rapoport J. L. (1997): Specific phobia, panic disorders, social phobia, and selective mutism. In: M. Wiener (Ed.): Textbook of Child and Adolescent Psychiatry, 2nd Edition. Washington: American Psychiatric Press, S. 491-506.

Black B.; Uhde T.W. (1992): Elective mutism as a variant of social phobia. In: Journal of the American Academy of Child \& Adolescent Psychiatry, 31(6), S. 1090-1094.

Black B.; Uhde T.W. (1994): Treatment of elective mutism with fluoxetine: A double-blind, placebocontrolled study. In: Journal of the American Academy of Child \& Adolescent Psychiatry, 33(7), S. 1000-1006.

Black B.; Uhde T.W. (1995): Psychiatric characteristics of children with selective mutism: A pilot study. In: Journal of the American Academy of Child \& Adolescent Psychiatry, 34(7), S. 847-856.

Böhm, W (2005): Wörterbuch der Pädagogik. Stuttgart: Alfred Kröner.

Bosman, D.B., Van der Merwe, I.W. \& Hiemstra, L.W. (2004). Tweetalige Woordeboek. AfrikaansEngels. Sewende Uitgawe. Tafelberg-Uitgewers.

Burbules, N.C. (1990): Varieties of Educational Dialogue. In: Philosophy of Education, S. 120-131.

Burbules, N.C. (1993): Dialogue in Teaching. Theory and practice. New York: Teachers College Press.

Burbules, N.C. (2003): Dialogue and Critical Pedagogy. In: Gur-Ze'ev, I. (Ed.): Critical Theory and Critical Pedagogy Today: Toward a New Critical Language in Education. Haifa: Faculty of Education. University of Haifa, S. 193 - 207.

Chevalley, Abel \& Marquerite (1980): The Concise French-English Dictionary. Oxford: Claendon Press. De Oliveira, P.C. (2006): Unperceived ideological transshipment and dialogue. Chapter IV - An 
example of the talismanic word: 'Dialogue'. http://www.tfp.org , Aufgerufen am 19. Mai 2006.

De Vos, A.S. (2002): Qualitative data analysis and interpretation. In: A.S. de Vos (Ed.): Research at grass roots. For the social sciences and human service professions (2nd ed.). Pretoria: Van Schaik, S. 339-355.

Department of Education (2007): Subject Assessment Guidelines Grades 10-12.

Department of Health and Human Services, Administration on Children, Youth and Families (2001).

Du Toit, P. (2006): Personal communication with the authors. Potchefstroom: North-West University.

Eksteen, L.C. (1984): Afrikaanse Sinoniemwoordeboek met antonieme. Pretoria: J.L. van Schaik.

Enoch, B. (2006): School violence reflection of society, not poor curriculum. In: The Herald, August 7, S. 8.

Ertmer, P. A. \& Newby, T.J. (1996): The Expert Learner: Strategic, self-regulated and reflective. In: Instructional Science. Vol. 24, S. 1 - 24.

Ertmer, P.A. (1997): Common qualitative research designs. In: P.D. Leedy: Practical research. Planning and Designing (6th ed.), New Jersey: Prentice-Hall, S. 155-172.

Fabbri, N. (2006): Community must assist schools in overcoming playground violence. In: Weekend Post, October 28, S. 6.

Finch, P. (Ed.). (s.a.): The new Elizabethan Reference Dictionary. Third Edition. London: George Newnes Ltd.

Foreman, J.B. (General Editor) (1975): Collins Contemporary Dictionary. London and Glasgow: Collins.

Freire, P. (1970): Cultural Action for Freedom. Cambridge, MA: Harvard Educational Review.

Geldenhuys, H. (2007): Mapping South Africa's crime hot spots. In: The Sunday Times, April, 22, S. 1.

Goosen, D. \& Rossouw, J. (2006): Gefrustreerde Begeertes. (Frustrierte Begierden.) In: Rapport, 5. November 2006, S. 23.

Groothoff, H.H. (1964): Pädagogik. Frankfurt am Main: Fischer Bücherei.

Hartle, R. (2006): Playground violence a wake-up call to 'deadbeat' absent parents. In: Weekend Post, 21. October 2006, S. 6.

Heidegger, M. (1977): Sein und Zeit. Frankfurt am Main: Vittorio Klostermann.

Hendricks, H.G. (1988): The Applied Principles of Learning Series. The 7 Laws of the Teacher. Course Notebook. Atlanta: Walk Thru the Bible Ministries, Inc.

Honderich, T. (Ed.) (2005): The Oxford Companion to Philosophy. Oxford: University Press.

http://en.wikipedia.org/wiki/Diagnostic_and_Statistical_Manual_of_Mental_Disorders

Jackson, D. zitiert in Kotzé, A. (2003): Onmoontlike kinders? Moenie vingers na skole wys. (Unmögliche Kinder? Schiebe die Schuld nicht auf die Schulen.). In: Rapport, 16. November 2003, S. 27.

Jaeger, R. M. (1988): Complementary Methods for Research in Education. Washington DC: American Educational Research Association.

Kirsten, G.J.C. (2006): Personal communication with the authors. Potchefstroom: North-West University.

Knutson, J.F., DeGarmo, D.S. \& Reid, J.B. (2004): Social Disadvantage and Neglectful Parenting as Precursors to the Development of Antisocial and Aggressive Child Behavior: Testing a Theoretical Model. In: Journal of Aggressive Behavior. Vol. 30, S. 187-205.

Kotzé, A. (2003): Onmoontlike kinders? Moenie vingers na skole wys. (Unmögliche Kinder? Schiebe die Schuld nicht auf die Schulen.). In: Rapport, 16. November 2004, S. 27.

Kritzinger, M.S.B. (1990): Handige Woordeboek. Afrikaans-Engels. English-Afrikaans. Pretoria: J.L. van Schaik.

Kritzinger, M.S.B., Schoeman, P.C. \& Cronjé, U.J. (1996): Groot Woordeboek. Afrikaans-Engels. Pretoria: J.L. van Schaik.

Langeveld, M.J. (1968): Beknopte Theoretische Pedagogik. 10th Edition. Groningen: Wolters- 
Noordhof.

Lefstein, A. (2006): Paper 33. Dialogue in Schools: Toward a Pragmatic Approach. In: Working Papers in Urban Language \& Literacies. London: Institute of Education.

Levinas, E. (1969): Het Menselijk Gelaat. Utrecht: Amboboeken.

Loeber, R. \& Stouthamer-Loeber, M. (1986): `Family Factors as correlates and predictors of juvenile conduct problems and delinquency'. In: M. Tonry and N. Morris (Eds.): Crime and Justice: An Annual Review of Research, vol. 7. Chicago: University of Chicago Press, S. 29-149.

Lombard, E. \& Fredericks, I. (2005): Violent pupils terrify teachers. In: Sunday Times, 20 March, p. 14.

Louw, L. (2006): SA polities fyn; veiligheid beroerd. (SA politically fine; safety wretched.). In: Die Burger, 7. November, 2006, S. S13).

Malan, M. \& Meyer, L. (2006): Ouers dra baie van die skuld. (Die Eltern tragen einen Großteil der Schuld.). In: Rapport, 29 October 2006, S. 4.

Malan, P. (2007): Geweld maak Suid-Afrika soos oorlogsland. In: Rapport, 3. Juni 2007, S. 18.

Microsoft ® Encarta ( Encyclopedie Winkler Prins (C) 1993-2004 Microsoft Corporation/Het Spectrum.

Middleton, J. R. \& Walsh, B. J. (1995): Truth is stranger than it used to be. Downer's Grove: InterVarsity Press.

Moffitt, T. E. (1990): 'The Neuropsychology of Juvenile Delinquency: A Critical Review'. In: M. Tonry and N. Morris (Eds.): Crime and Justice: An Annual Review of Research. Vol. 12. Chicago: University of Chicago Press, S. 99-169.

Nienhuis, J. (2006): Heropvoedingsindustrie bloeit in VS. In: AD Zaterdag, September, S. 27.

Olojede, D (2006): This is how the rot begins. In: Sunday Times, November 5, p. 41.

Orr, D. (1991): What Is Education For? Six myths about the foundations of modern education, and six new principles to replace them. In: Annals of Earth. Vol. VIII, No. 2, pp. 52-65.

Postma, F. 1992. Beknopte Woordeboek. Latyn-Afrikaans. Pretoria: H.A.U.M.

Potgieter, F.J. (1991): Die Opleiding van Afrikataalonderwysers vir die Primêre Skool. ' $n$ Studie in Tydsperspektief. Unpublished DEd-thesis. Pretoria. University of South-Africa (UNISA).

Potgieter, F.J. (2004): Transcription of focus-group interview with two classroom educators and three secondary school principals. Pretoria: University of Pretoria.

Potgieter, F.J. (2006): Mentoring school-based educators-in-training. Short course of the Faculty of Education Sciences. Potchefstroom: North-West University.

Pretorius, J.W.M. (1999): Opvoeding, Samelewing, Jeug - 'n Sosiopedagogiek-leerboek. Pretoria: Van Schaik Publishers.

Punch, K. (1998): Introduction to social research. Quantitative and qualitative approaches. London: SAGE.

Rockwell, G.. 2003. Defining Dialogue: From Socrates to the Internet. Amherst, New York: Humanity Books.

Rossouw, J. P. (2007): The effect of learner discipline on educator security - an international qualitative survey. In: Oosthuizen, I. J., Rossouw, J. P., Russo, C., Van der Walt, J. L. and Wolhuter, C. C.: Perspectives on learner conduct. Proceedings of the first international Conference on Learner Discipline at the North-West University, Potchefstroom Campus, 2.-4. April 2007, S. 212-227.

Rottschaefer, W.A. (2000): Naturalizing ethics: the Biology and Psychology of Moral Agency. In: Zygon. Vol. 35, No. 2, S. 253-286.

Russo, C. J. (2007): Student discipline in American schools. In: Oosthuizen, I. J., Rossouw, J. P., Russo, C., Van der Walt, J. L. and Wolhuter, C. C.: Perspectives on learner conduct. Proceedings of the first international Conference on Learner Discipline at the North-West University. Potchefstroom Campus, 2.-4. April 2007, S. 228-242.

Schipper, M. (2001): Nederlandse kinderen de ergste! In: De Telegraaf, 15. September 2001, S. T19. 
Schoonees, P.C., Swanepoel, C.J., Du Toit, S.J. \& Booysen, C.M. (1975): HAT Verklarende Handwoordeboek van die Afrikaanse Taal. Pretoria: Voortrekkerpers.

Seleoane, M. 2006. Police still in quandary as crime wave carries on. In: The Herald, 6. Juli 2006, S. 4. Seton-Smith, C. (2004): Who's to blame for our troubled world? In: Algoa Sun, 16. Dezember 2004., S. 16.

Simpson, D.P. (1980): Cassell's New Latin Dictionary. Third Edition. London: Cassell.

Slade, J. \& Schneider, J. (2001): Disruptive and Anti-Social Behaviour Report: Antisocial behaviour in Darlington - prevalence and treatment. Durham: Centre for Applied Social Studies. University of Durham.

Smit, A.J. (1977): Taalfenomenologie in Pedagogiese Perspektief. Unpublished DEd-thesis. Pretoria: University of South Africa.

Snyman, M. (2007): Crime reporting in the press. In: Zeitschrift für die Geisteswissenschaften, 47(4), S. $103-118$.

Staude, B. (2007): Young people's behaviour reflects wider community. In: The Herald, 25. April 2007, S. 7.

Steyn, M. M. \& Strydom, H. (2004): Misdaad en die impak daarvan op die mens - 'n Christelike perspektief. In: Koers 69(2), S. 337-358.

Strelen, B. \& Gonin, H.L. (1970): Woordeboek. Afrikaans-Frans, Frans-Afrikaans. Pretoria: J.L. van Schaik.

Sutherland, E.H. \& Cressey, D.R. (1970): Criminology. Philadelphia: Lippencott.

Sykes, J.B. (Ed.) (1993): The Concise Oxford Dictionary. Oxford: Clarendon Press.

Ten Have, T.T. (1970): Klein bestek van de agologie en van de studie der agologie. Groningen: WoltersNoordhoff.

Ten Have, T.T. (1973): Andragogie in Blauwdruk. Groningen: H.D. Tjeenk-Willink.

Ten Have, T.T. (s.a.): Pedagogie en andragogie in onderlinge verhouding. In: Pedagogiek in ontwikkeling. Th. G. Bolleman (Ed.). Uitgeverij Zwijsen.

Terblanche, H.J. \& Odendaal, J.J. (1976): Afrikaanse Woordeboek, Verklarend met Woordafleidings. Johannesburg: Afrikaanse PersBoekhandel.

The Oxford English Dictionary (1993 a). Volume III. Oxford: Clarendon Press.

The Oxford English Dictionary. (1993 b). Volume VI. Oxford: Clarendon Press.

Thompson, A. (2004): SA not a safe place, despite crime drop. In: The Herald, 22. September 2004, S. 11.

Thomson, D. (2007): Genoeg van misdaadmoeras. In: Die Burger, 19. April 2007, S. 11.

Thornberry, T. P. (Ed.) (1977): Advances in Criminological Theory. Vol. 7: Developmental Theories of Crime and Delinquency. New Brunswick and London: Transaction, S. 11-54

U.S. Department of Health and Human Services, Administration for Children and Families, Administration on Children, Youth and Families, Children's Bureau (2001). Child welfare outcomes 2001: Annual report. Washington, DC: U.S. Government Printing Office.

Van der Walt, J.L. \& Potgieter, F.J. (2006): REDCo-DIALOG Project Research Method: Final Draft. Port Elizabeth. 29. Mai 2006.

Van Hijum, E. (2006): Opvoedkamp nodig als stok achter de deur. In: AD Zaterdag, 26. September 2006, S. 26.

Van Niekerk, P. (2006): Rapists weep after sentencing. In: The Herald, 6. Juli 2006, S. 4.

Van Rensburg, C.J.J. \& Landman, W.A. (1995): Notes on Fundamental Pedagogic Concepts. Goodwood: NG Kerk Boekhandel.

Verbrugge, A. (2004): Tijd van onbehagen. Amsterdam: SUN.

Wolters, A. M. (1992): Die skepping herwin. Potchefstroom: IRS. 


\section{Über die Autoren}

Prof. Dr. Ferdinand Potgieter, Professor für Philosophische Pädagogik, Nordwest-Universität, Südafrika. Kontakt: Ferdinand.Potgieter@nwu.ac.za

Prof. Dr. Hannes van der Walt, Professor für Philosophische Pädagogik, Nordwest-Universität, Südafrika. Kontakt: jlvdwalt@intekom.co.za

Prof. Dr. Charl Wolhuter, Professor für Vergleichende und Internationale Pädagogik, NordwestUniversität, Südafrika. Kontakt: Charl.Wolhuter@nwu.ac.za

1 Siehe dazu Carey und Goldman (in Stoff [Hg.], 1997, 243-254) zu den genetischen Einflüssen auf antisoziales Verhalten sowie Moffitt (1990, 99-169) zu dem Einfluss von Faktoren, die mit dem zentralen Nervensystem zusammenhängen. Lombroso (1835-1909) entwickelte die biologische Kriminalitätstheorie, die gesetzwidriges Verhalten über genetisch/biologische Eigenschaften erklärt. Die Milieutheorien der französischen kriminalsoziologischen Schule (vor allem A. Lacassagne [1843-1924]) entstanden als Gegenlehre zu den von Lombroso vertretenen Theorien. Durkheim (1858-1917) setzte kriminelles Verhalten in einer Gesellschaft als verhältnismäßig 'normal' voraus. Seiner Meinung nach sind die Widersprüche in einer besonderen Gesellschaft, die wiederum auf Interessenskollisionen zurückzuführen sind, die eigentlichen Ursachen kriminellen Verhaltens. Inspiriert von Durkheim entwickelte Merton die Anomietheorie, die auf der Idee basiert, dass es in einer besonderen Gesellschaft immer Diskrepanzen zwischen den verschiedenen kulturellen Zielen, die für bestimmte Individuen bzw. Gruppen wichtig sind, und den Mitteln, solche Ziele zu erreichen, gibt. Nach der differentiellen Theorie der Assoziation von Edwin H. (Sutherland \& Cressey, 1970) kann kriminelles Verhalten während Interaktionen und Kommunikationsprozessen mit anderen Personen erlernt werden (Microsoft Encarta Winkler Prins, 1993-2004). Die von Thornberry herausgegebene Aufsatzreihe (1977, 11-54) behandelt die wichtigsten theoretischen Traditionen. Loeber und Stouthamer-Loeber $(1986,29-149)$ erforschten die Theorie des sogenannten 'Familienfaktors'. 